\title{
Air and Bone Conduction Frequency-specific Auditory Brainstem Response in Children with Agenesis of the External Auditory Canal
}

\author{
Pricila Sleifer ${ }^{1}$ \\ Rudimar dos Santos Riesgo ${ }^{3}$ \\ ${ }^{1}$ Department of Human Health and Communication, Universidade \\ Federal do Rio Grande do Sul, Porto Alegre, Rio Grande do Sul, Brazil \\ ${ }^{2}$ Child and Adolescent Health Program, Universidade Federal do Rio \\ Grande do Sul Ringgold Standard Institution, Porto Alegre, \\ Rio Grande do Sul, Brazil \\ ${ }^{3}$ Child Neurology Unit (HCPA-UFRGS), Porto Alegre, Rio Grande do Sul, \\ Brazil
}

Int Arch Otorhinolaryngol 2017;21:318-322.

\author{
Address for correspondence Pricila Sleifer, PhD, Child and Adolescent \\ Health, Department of Human Health and Communication, \\ Universidade Federal do Rio Grande do Sul, Ramiro Barcelos, 2600 \\ Porto Alegre, Rio Grande do Sul, Brazil \\ (e-mail: pricilasleifer@uol.com.br).
}

\begin{abstract}
Introduction The tone-evoked auditory brainstem responses (tone-ABR) enable the differential diagnosis in the evaluation of children until 12 months of age, including those with external and/or middle ear malformations. The use of auditory stimuli with frequency specificity by air and bone conduction allows characterization of hearing profile.

Objective The objective of our study was to compare the results obtained in tone-ABR by air and bone conduction in children until 12 months, with agenesis of the external auditory canal.

Method The study was cross-sectional, observational, individual, and contemporary. We conducted the research with tone-ABR by air and bone conduction in the frequencies of $500 \mathrm{~Hz}$ and $2000 \mathrm{~Hz}$ in 32 children, 23 boys, from one to 12 months

Keywords

- evoked potentials

- auditory

- hearing loss

- conductive

- bone conduction

- congenital abnormalities

- ear

- external old, with agenesis of the external auditory canal.

Results The tone-ABR thresholds were significantly elevated for air conduction in the frequencies of $500 \mathrm{~Hz}$ and $2000 \mathrm{~Hz}$, while the thresholds of bone conduction had normal values in both ears. We found no statistically significant difference between genders and ears for most of the comparisons.

Conclusion The thresholds obtained by bone conduction did not alter the thresholds in children with conductive hearing loss. However, the conductive hearing loss alter all thresholds by air conduction. The tone-ABR by bone conduction is an important tool for assessing cochlear integrity in children with agenesis of the external auditory canal under 12 months.
\end{abstract}

\section{Introduction}

The early identification of hearing loss is essential for the appropriate language and cognitive development of children.
The audiological diagnosis must be done by the critical language acquisition period to achieve the maximum neural plasticity. The advances of technological have allowed an objective assessment of hearing, which is essential for received

June 22, 2016

accepted

December 21, 2016

published online

February 13, 2017
DOI https://doi.org/

10.1055/s-0037-1598243. ISSN $1809-9777$.
Copyright $(2017$ by Thieme Revinter

Publicações Ltda, Rio de Janeiro, Brazil
License terms

(c) (i) $\ominus \circledast$ 
children who are not able to respond the audiological behavioral assessment.

The tone-evoked auditory brainstem response (tone-ABR) is considered the gold standard to determine electrophysiological thresholds in children younger than 24 moths. ${ }^{1-13}$ The tone-ABR can be performed by air and bone conduction, in the frequencies of 500 to $4000 \mathrm{~Hz}$, which allows the comparison of the electrophysiological thresholds and the hearing loss classification. . $8,10,11,13$ There is an air-bone gap in conductive hearing loss, which are greater or equal than $15 \mathrm{~dB}$ (decibel). This gap is not observed in sensorioneural hearing loss. $2,3,8,14$

The tone-ABR assessment by bone conduction is essential for diagnosis in young children with external or middle ear malformations. The electrophysiological thresholds by air and bone conduction allow the characterization of the audiological profile, ${ }^{2,4,9,12,15-18}$ which have a good correlation with the behavioral audiological assessment. ${ }^{7}$

Some researches with tone-ABR in children with ear malformation ${ }^{4,13,16,19,20}$ demonstrated the higher thresholds by air assessment than bone conduction, which characterizes the conductive hearing loss, commonly associated with the ear malformations.

The agenesis of the external auditory canal is an important ear malformation characterized by absence of external auditory canal, which may affect the right and/or the left ear. ${ }^{18,21-23}$ Changes in the middle ear, as the absence of the tympanic membrane, can also be associated with agenesis of the external auditory canal. This can be explained by the same embryological origin of the structures.

Despite the importance of the early audiological assessment by the tone-ABR bone conduction in children under 12 months with agenesis of the external auditory canal, we have not found current articles on this topic. This can be explained by the low prevalence of this ear malformation, ${ }^{24}$ that is, 1 in 10.000 to 20.000 live births. ${ }^{25}$ Furthermore, in many cases, the audiological assessment by tone-ABR by bone conduction is not a routine in the audiological diagnosis and this can compromise the correct audiological profile.

Thus, the purpose of this research was to analyze the electrophysiological thresholds by tone-ABR by air and bone conduction in children under 12 months of age with agenesis of the external auditory canal.

\section{Methods}

This research is an observational, cross-sectional, individual, and contemporary study with children from a hospital. The sample consisted of non-probabilistic and convenience. The Scientific Committee and Research Ethics Committee evaluated and approved the project (protocol $n^{\circ} 2011039$.). Still emphasizing the completeness of Resolution 466/12 which deals with human research, only the children whose parents or guardians signed the Informed Consent participated of this study.

The research included 32 term children, aged between one and 12 months, of both genders, who had right and/or left agenesis of the external auditory canal. The diagnosis of malformation was performed by a medical evaluation, where children are accompanied.
All children have the hearing assessment by the tone-ABR by air and bone conduction. For the children with unilateral agenesis of the external auditory canal, the electrophysiological thresholds were obtained for both ears. However, for this research we considered only in the thresholds by the ear with malformation.

All children remained in natural sleep and were positioned comfortably in the mother's lap or caregiver in a reclining chair.

The tone-ABR was conducted with the equipment Smart EP, with two channels, Intelligent Hearing Systems (IHS) brand. The thresholds were obtained by air and bone conduction, sequentially, through supra-aural TDH-39 and bone vibrator B-71 Radio EAR in the upper posterior auricular position. The bone vibrator was fixed with a self-adherent elastic wrap. ${ }^{2} \mathrm{We}$ placed the reference electrodes on the right (M2) and left (M1) mastoid, and the active $(\mathrm{Fz})$ and ground $(\mathrm{Fpz})$ on the forehead. We cleaned the skin with gauze and Nuprep, to reduce electrical impedance between the skin and the electrode. We maintained impedance at or below 3 Kohms.

We obtained the electrophysiological thresholds for the frequencies of 500 and $2000 \mathrm{~Hz}$. The results were considered normal when the electrophysiological thresholds were less or equal to 30 and $35 \mathrm{dBNa}$ by air and less or equal to 20 and 30dBNA by bone conduction, for 500 and $2000 \mathrm{~Hz}$, respectively. ${ }^{7}$ The frequency of $2000 \mathrm{~Hz}$ was the first to be tested. The electrophysiological thresholds were identified by the wave $\mathrm{V}$ in both transducers. The initial intensity was $80 \mathrm{dBnNA}$ for air and 50 $\mathrm{dBnHL}$ for bone conduction, being decreased from 10 to $10 \mathrm{~dB}$ until the wave $V$ was not detected. From this, the intensity was increased from 10 to $10 \mathrm{~dB}$ to determine in which intensity the wave $\mathrm{V}$ was detected. The contralateral air masking was used with intensity by $40 \mathrm{~dB}^{4}$ for the children with unilateral agenesis of external auditory canal. We reapplied electrophysiological threshold by air and bone conduction in all intensities to check the reproducibility of wave $\mathrm{V}$. We used a minimum of 2,000 sweeps to obtain each electrophysiological threshold. The protocol used in this research is described in - Table $\mathbf{1}$.

We created the database on the Excel program and analyzed the data with SPSS (Statistical Package for Social Sciences) version 20.0. We describe continuous variables as median, standard deviation, minimum, and maximum and present the categorical variables by absolute and relative frequencies. We used the Wilcoxon test to compare the electrophysiological thresholds between air and bone conduction and between frequencies. We used the Mann-Whitney test to compare genders. The level of statistical significance was $5 \%$ ( $\mathrm{p} \leq 0.05$ ) for all statistical tests.

\section{Results}

For this research 32 children with agenesis of the external auditory canal were evaluated by tone-ABR, 23 males (71.8\%) and 9 females (28.1\%), aged between one and 12 months (-Table 2).

The electrophysiological thresholds by air conduction were statistically significant higher than bone conduction in both ears and in both tested frequencies ( - Table 3 ). 
Air and Bone Conduction Frequency-specific Auditory Brainstem Response in Children with Agenesis of the External

Table 1 Tone-ABR parameters by air and bone conduction for 500 and $2000 \mathrm{~Hz}$

\begin{tabular}{|l|l|l|}
\hline & Tone-ABR by air conduction & Tone-ABR by bone conduction \\
\hline Transducer & Supra-aural TDH-39 & Bone vibrator B-71 Radio EAR \\
\hline Stimulus & Tone burst $(500$ and $2000 \mathrm{~Hz})$ & Tone burst $(500$ and $2000 \mathrm{~Hz})$ \\
\hline Polarity & Alternating & Alternating \\
\hline Sweeps & 2000 to 4000 & 2000 to 4000 \\
\hline Rate & $37.1 / \mathrm{s}($ sweeps per second) & $37.1 / \mathrm{s}$ \\
\hline Filters & $100-3000 \mathrm{~Hz}$ & $100-3000 \mathrm{~Hz}$ \\
\hline Analysis time window & $25 \mathrm{~ms}$ & $25 \mathrm{~ms}$ \\
\hline Envelope & Blackman & Blackman \\
\hline Signal amplification & 100.000 & 100.000 \\
\hline Masking & not applicable & Contralateral; Intensity: $40 \mathrm{~dB}$ \\
\hline Impedance & Less than 5 Kohms & Less than 5 Kohms \\
\hline
\end{tabular}

There was no statistically significant difference between the ears and gender for the two tested frequencies in the comparison between air and bone conduction for most of the comparisons (- Tables 3, 4, and 5).

\section{Discussion}

The electrophysiological hearing assessment in children under 12 months of age allows the precise identification of hearing loss. ${ }^{9,11,13,16-18,26,27}$ Research by bone conduction is essential in children with ear malformations.

The craniofacial malformations are associated with changes in the fetal development of the pharyngeal arches, the development of which depends on complex interactions between the ectoderm, endoderm, and mesoderm, along with the neural crest that populates each $\operatorname{arch} .^{28}$ In this research, agenesis of the external auditory canal had higher prevalence in boys (71.8\%), in agreement with other authors. ${ }^{27}$ In addition, there was a higher occurrence of unilateral agenesis (84.3\%) than bilateral. These findings are similar to other studies in children with ear malformations. $^{21,22,24,25,27-29}$ On the other hand, some researchers ${ }^{23}$ did not report predominance of unilateral malformation.

Table 2 Descriptive statistic of the children with agenesis of the auditory canal

\begin{tabular}{|l|c|}
\hline & $\mathbf{n}=32(\%)$ \\
\hline $\begin{array}{l}\text { Age (months) - Median } \\
\text { (min - max) }\end{array}$ & $6(1-12)$ \\
\hline Gender - n (\%) & $23(71.87)$ \\
\hline Male & $9(28.13)$ \\
\hline Female & $17(53.12)$ \\
\hline Affected ear - n (\%) & $10(31.25)$ \\
\hline Right & $5(15.62)$ \\
\hline Left &
\end{tabular}

Abbreviations: min, minimum value; max, maximum value.
In this study, we found that the agenesis of the external auditory canal had a greater involvement on the right ear (53.12\%), followed by left ear (31.25\%), and bilateral (15.62\%) ear malformation. Our results agree with other studies. ${ }^{21,22,24}$

For the comparison of air and bone conduction of tone$\mathrm{ABR}$, this study showed higher air electrophysiological thresholds to 500 and $2000 \mathrm{~Hz}$ than the bone electrophysiological thresholds to the same frequencies. These findings corroborate with other studies. $^{14,30-32}$ In addition, the bone thresholds remained within the mean for the normal criteria, confirming the conductive hearing loss in children with agenesis of the external auditory canal. These results are important for the children to benefit from appropriate treatment, reducing the damage caused by hearing loss. ${ }^{10,25,33}$ Thus, the audiological assessment with tone-ABR by bone conduction in children under 12 months is essential to the early identification of conductive hearing loss to achieve the maximum neural plasticity with the correct treatment.

In this study, there was no statistically significant difference between most comparisons of tone-ABR in the ears with agenesis of the external auditory canal both by air and by bone conduction research. These findings corroborate with the results reported by other studies, ${ }^{5,21,34,35}$ but we found no studies with comparisons between ears in children

Table 3 Comparison between tone-ABR thresholds by air and bone conduction

\begin{tabular}{|l|c|c|c|c|}
\hline Frequency & Ear & $\begin{array}{c}\text { Bone md } \\
(\mathbf{m i n}-\mathbf{m a x}) \\
(\mathrm{dBHL})\end{array}$ & $\begin{array}{c}\text { Air md } \\
(\mathbf{m i n}-\mathbf{m a x}) \\
(\mathbf{d B H L})\end{array}$ & $\mathrm{p}^{*}$ \\
\hline $500 \mathrm{~Hz}$ & Right & $25(20-30)$ & $55(40-70)$ & $0.001^{*}$ \\
\hline & Left & $20(20-30)$ & $50(40-70)$ & $0.001^{*}$ \\
\cline { 3 - 4 } & & $p=0.655^{*}$ & $p=0.038^{*}$ & \\
\hline $2000 \mathrm{~Hz}$ & Right & $25(20-30)$ & $50(30-70)$ & $0.001^{*}$ \\
\hline & \multirow{2}{*}{ Left } & $20(20-25)$ & $45(30-70)$ & \multirow{2}{*}{$0.001^{*}$} \\
\cline { 3 - 4 } & & $p=1.00^{*}$ & $p=0.655^{*}$ & \\
\hline
\end{tabular}

Abbreviations: $\mathrm{dBHL}$, decibel hearing level; md, median; min, minimum value; max, maximum value.

*Wilcoxon Test. 
Table 4 Comparison between frequencies of tone-ABR thresholds by air and bone conduction

\begin{tabular}{|l|c|c|c|c|}
\hline & Ear & $\begin{array}{c}500 \mathrm{~Hz} \text { md } \\
(\mathrm{min}-\mathrm{max}) \\
(\mathrm{dBHL})\end{array}$ & $\begin{array}{c}2000 \mathrm{~Hz} \text { md } \\
(\mathbf{m i n}-\mathrm{max}) \\
(\mathrm{dBHL})\end{array}$ & \\
\hline Air & Right & $55(40-70)$ & $50(30-70)$ & $0.002^{*}$ \\
\hline \multirow{2}{*}{ Bone } & Left & $50(40-70)$ & $45(30-70)$ & \multirow{2}{*}{$0.004^{*}$} \\
\cline { 3 - 4 } & Right & $25(20-30)$ & $25(20-30)$ & \multirow{2}{*}{$0.785^{*}$} \\
\hline \multirow{2}{*}{} & Left & $20(20-30)$ & $20(20-25)$ & \multirow{2}{*}{$0.059^{*}$} \\
\cline { 3 - 4 } & & $p=0.655^{*}$ & $p=1.00^{*}$ & \\
\hline
\end{tabular}

Abbreviation: $\mathrm{dBHL}$, decibel hearing level; md, median; min, minimum value; max, maximum value.

*Wilcoxon Test.

with agenesis of the external auditory canal. In this research the frequency of $500 \mathrm{~Hz}$ in the right ear by air conduction had higher thresholds than the left ear. This results disagrees with other researches with hearing electrophysiology. ${ }^{5,21,34,35}$ However, the results from this study can be justified by the number of ears with agenesis of the external auditory canal which is greater on the right ear and may have influenced the ranking of data analyzed by the Wilcoxon test. Analyzing the literature, it is clear that, despite the comparison between ears be important, many studies assess the bone electrophysiological thresholds only in one ear, limiting the comparison of the results from this study. In this study, we assert that the comparison between ears can contribute to future research comparing the degree of hearing loss between ears in children with agenesis of the external auditory canal.

In this study, there was no statistically significant difference between genders for the tone-ABR by bone conduction, agreeing with others studies. ${ }^{5,8,21}$ In contrast, our results disagree with a study, ${ }^{34}$ which found higher tone-ABR thresholds in the female than male gender. In the present study, boys had higher thresholds by air in the right ear than girls. ${ }^{5}$ The high incidence of the agenesis of the external auditory canal in males compared with females may have influenced this result.
The results from this study showed values of the electrophysiological thresholds by air tone-ABR of $500 \mathrm{~Hz}$ higher than $2000 \mathrm{~Hz}$. This finding agree with others research, 5,32,35 in which the authors reported that the frequency of $500 \mathrm{~Hz}$ had a higher threshold when compared with higher frequencies. This fact can be explained by the difficulty of the identification of wave $\mathrm{V}$ in lower frequencies, mainly in lower intensities. On the other hand, the audiometric configuration in conductive hearing loss show worse results in lower frequencies, which is compatible with this type of hearing loss.

Overall, the results of this study are in agreement with other studies with tone-ABR bone conduction. 1,7,27,30,31 However, there is a large number of studies that use tone-ABR by air only in the national literature. The lack of precision of the protocols used in the tone-ABR by bone conduction can hinder the classification of the normal criteria and, consequently, the use of this type of assessment. ${ }^{10,19,30}$ In this research, we only evaluated the thresholds of the 500 and $2000 \mathrm{~Hz}$ due to the protocols in the scientific literature $e^{1,5,7,8}$ found prior the data collection period. However, most recent research ${ }^{10}$ proposed the normality criteria for the frequencies of 500, 1000, 2000, and $4000 \mathrm{~Hz}$ in neonates, which can facilitate the classification of normal or hearing loss for this age group.

This research found normal electrophysiological thresholds obtained by tone-ABR bone conduction in contrast with abnormal results by air tone-ABR assessment. These results showed the conductive hearing loss in all tested children. The assessment with tone-ABR bone conduction is an important tool to assess the cochlear integrity in children under 12 months of age with agenesis of the auditory canal, allowing the identification of the type and the degree of hearing loss for early intervention.

\section{Conclusion}

The results of tone-ABR in children with agenesis of the auditory canal showed higher thresholds by air than bone conduction. The electrophysiological thresholds of tone-ABR by bone conduction were normal for both tested frequencies. In addition, the variables gender and ear did not influence in the most electrophysiological responses.

Table 5 Comparison between genders

\begin{tabular}{|c|c|c|c|c|c|}
\hline & Ear & Frequencies & $\begin{array}{c}\text { Male md } \\
(\min -\max ) \\
(\mathrm{dBHL})\end{array}$ & $\begin{array}{c}\text { Female md } \\
(\min -\max ) \\
(\mathrm{dBHL})\end{array}$ & $\mathrm{p}^{*}$ \\
\hline \multirow[t]{4}{*}{ Air } & \multirow[t]{2}{*}{ Right } & $500 \mathrm{~Hz}$ & $60(45-70)$ & $42.5(40-50)$ & $0.004^{*}$ \\
\hline & & $2000 \mathrm{~Hz}$ & $50(45-50)$ & $40(40-40)$ & $0.004^{*}$ \\
\hline & \multirow[t]{2}{*}{ Left } & $500 \mathrm{~Hz}$ & $57.5(40-70)$ & $50(40-50)$ & $0.129^{*}$ \\
\hline & & $2000 \mathrm{~Hz}$ & $50(30-70)$ & $40(30-50)$ & $0.254^{*}$ \\
\hline \multirow[t]{4}{*}{ Bone } & \multirow[t]{2}{*}{ Right } & $500 \mathrm{~Hz}$ & $25(20-30)$ & $20(20-30)$ & $0.275^{*}$ \\
\hline & & $2000 \mathrm{~Hz}$ & $25(20-30)$ & $25(20-25)$ & $0.897^{*}$ \\
\hline & \multirow[t]{2}{*}{ Left } & $500 \mathrm{~Hz}$ & $22.5(20-30)$ & $20(20-30)$ & $0.440^{*}$ \\
\hline & & $2000 \mathrm{~Hz}$ & $20(20-25)$ & $20(20-20)$ & $0.371^{*}$ \\
\hline
\end{tabular}

Abbreviations: $\mathrm{dBHL}$, decibel hearing level; md, median; min, minimum value; max, maximum value.

* Mann-Whitney Test. 
The tone-ABR by air and bone conduction in children with agenesis of the external auditory canal is an important tool to the correct audiological diagnosis, assisting in appropriate early intervention.

\section{References}

1 Stapells DR. Threshold Estimation by the Tone-Evoked Auditory Brainstem Response: A Literature Meta-Analysis. J Speech Lang Pathol Audiol2000b; 24(02):74-83

2 Chapchap MJ. [Dissertation]. Respostas elétricas de tronco encefálico por estimulação aérea e óssea em neonatos. São Paulo, Brazil: Escola Paulista de Medicina, Universidade Federal de São Paulo; 2002

3 American Academy of Pediatrics, Joint Committee on Infant Hearing. Year 2007 position statement: Principles and guidelines for early hearing detection and intervention programs. Pediatrics 2007;120(04):898-921

4 Alvarenga KF, Araújo ES. Avaliação audiológica de 0 a 1 ano de idade. In: Boéchat EM, Menezes PL, Couto CM, Frizzo ACF, Scharlach RC. Anastásio ART. Tratado de Audiologia. 2nd Ed. São Paulo: Santos; 2015:395-406

5 Almeida MG, Rodrigues GRI, Lewis DR. Potenciais evocados auditivos por frequência específica em lactentes com audição normal. Rev CEFAC 2011;13:489-495

6 Farias VB, Sleifer P, Pauletti LF, Krimberg CFD. Correlation of the findings of auditory steady-state evoked potential and of behavioral hearing assessment in infants with sensorineural hearing loss. CoDAS 2014;26(03):226-230

7 Stapells DR. Frequency-specific threshold assessment in young infants using the transient ABR and the brainstem ASSR. In: Seewal RC, Tharp AM. Comprehensive handbook of pediatric audiology. San Diego: Plural Publishing; 2011:409-48

8 Matas CG, Haitaima NM, Gonçalves IC. Estabilidade dos potenciais evocados auditivos em indivíduos adultos com audição normal Rev Soc Bras Fonoaudiol 2011;16(01):37-41

9 British Columbia Early Hearing Program (BCEHP). Audiology Assessment Protocol. Version 4.1. 2012; available at:http://www. phsa.ca/Documents/bcehpaudiologyassessmentprotocol.pdf

10 Ramos N, Lewis DR. Potencial Evocado Auditivo de Tronco Encefálico por frequência específica por via aérea e via óssea em neonatos ouvintes normais. Rev CEFAC 2014;16(03):757-767

11 Zhang Y, Fan Y, Wang Y, Chen X. [Efficacy of BAHA softband in young children with bilateral congenital aural atresia]. Zhonghua Yi Xue Za Zhi 2014;94(06):420-423

12 Sleifer P. Avaliação eletrofisiológica da audição em crianças. In: Cardoso MC. Fonoaudiologia na infância: avaliação e tratamento. Rio de Janeiro: Revinter; 2015:171-94

13 Liang S, Soli SD, Zheng Y, Li G, Meng Z. Initial classification of pediatric hearing impairment using behavioral measures of early prelingual auditory development. Int J Audiol 2016;55(04):224-231

14 Gomes DT. [Dissertação de Mestrado]. Potencial Evocado Auditivo por Condução Óssea em Pacientes com Microssomia Craniofacial. Curitiba, Brazil: Universidade Tuiuti do Paraná; 2007

15 Rodrigues GRI, Almeida MG, Lewis DR. Potenciais evocados auditivos de tronco encefálico por frequência específica e de estado estável na audiologia pediátrica: estudo de caso. Rev Soc Bras Fonoaudiol 2009;14(04):534-538

16 Hatton JL, Janssen RM, Stapells DR. Auditory brainstem responses to bone-conducted brief tones in young children with conductive or sensorineural hearing loss. Int J Otolaryngol 2012;2012(12):284864
17 Tekes A, Ishman SL, Baugher KM, et al. Does microtia predict severity of temporal bone CT abnormalities in children with persistent conductive hearing loss? J Neuroradiol 2013;40(03):192-197

18 Dougherty W, Kesser BW. Management of Conductive Hearing Loss in Children. Otolaryngol Clin North Am 2015;48(06):955-974

19 Curado NRPV, Muniz LF, Silveira AK, Silva ARA, Griz SMS. Potencial evocado auditivo de tronco encefálico por condução óssea: uma revisão integrativa. Rev CEFAC 2015;17(02):635-647

20 Anwer C, Schwarz T, Volk SW, Vite C. BAER testing in a dog with bilateral external ear canal atresia. J Am Anim Hosp Assoc 2011; 47(05):370-374

21 Bartel-Friedrich S, Wulke C. Classification and diagnosis of ear malformations. GMS Curr Top Otorhinolaryngol Head Neck Surg 2007;6:Doc05

22 Bartel-Friedrich S. Congenital Auricular Malformations: Description of Anomalies and Syndromes. Facial Plast Surg 2015;31(06):567-580

23 Paccola ECM, Fernandes JC, Mondelli MFCG. Amplificação por condução óssea em malformações congênitas: benefício e satisfação. Rev Bras Otorrinolaringol (Engl Ed) 2013;79(03):359-365

24 White RD, Ananthakrishnan G, McKean SA, Brunton JN, Hussain SSM, Sudarshan TA. Masses and disease entities of the external auditory canal: radiological and clinical correlation. Clin Radiol 2012;67(02):172-181

25 Sanhueza CD, Esquivel CP, Dentone SC. Review of the management of external auditory canal atresia using Osseo integrated implantable hearing devices: Report of a successful case with Bonebridge ${ }^{\circledR}$ implant. Rev Otorinolaringol Cir Cabeza Cuello 2014; 74(02):161-166

26 Valenzuela DG, Kumar DS, Atkins CL, Beers A, Kozak FK, Chadha NK. Chloral hydrate sedation for auditory brainstem response (ABR) testing in children: Safety and effectiveness. Int J Pediatr Otorhinolaryngol 2016;83:175-17810.1016/j.jporl.2016.02.006

27 Romero ACL, Delecrode CR, Cardoso ACV, Frizzo ACF. Potencial evocado auditivo de tronco encefálico em crianças encaminhadas de um programa de triagem auditiva neonatal. Rev Bras Saude Mater Infant 2012;12(02):145-153

28 Tassano E, Jagannathan V, Drögemüller C, et al. Congenital aural atresia associated with agenesis of internal carotid artery in a girl with a FOXI3 deletion. Am J Med Genet A 2015;167A(03):537-544

29 Wei J, Ran S, Yang Z, Lin Y, Tang J, Ran H. Prenatal ultrasound screening for external ear abnormality in the fetuses. [Published online June, 2014, 2014]BioMed Res Int 2014;2014:35756410.1155/2014/357564

30 Fichino SN, Lewis DR, Fávero ML. Electrophysiologic threshold study in air and bone conduction in children with 2 months or less age. Rev Bras Otorrinolaringol 2007;73(02):251-256

31 Gorga MP, Kaminski JR, Beauchaine KL, Bergman BM. A comparison of auditory brain stem response thresholds and latencies elicited by air- and bone-conducted stimuli. Ear Hear 1993;14(02):85-94

32 Garcia MV, Azevedo MF, Biaggio EPV, Didoné DD, Testa JRG. Potencial evocado auditivo de estado estável por via aérea e via óssea em crianças de zero a seis meses sem e com comprometimento condutivo. Rev CEFAC 2014;16(03):699-706

33 Nadaraja GS, Gurgel RK, Kim J, Chang KW. Hearing outcomes of atresia surgery versus osseointegrated bone conduction device in patients with congenital aural atresia: a systematic review. Otol Neurotol 2013;34(08):1394-1399

34 Cone-Wesson B, Ramirez GM. Hearing sensitivity in newborns estimated from ABRs to bone-conducted sounds. J Am Acad Audiol 1997;8(05):299-307

35 Elsayed AM, Hunter LL, Keefe DH, et al. Air and Bone Conduction Click and Tone-Burst Auditory Brainstem Thresholds Using Kalman Adaptive Processing in Nonsedated Normal-Hearing Infants. Ear Hear 2015;36(04):471-481 